\title{
The Practical Method and Experimental Verification of Temperature Estimation in the Permanent Magnet of Electric Machine
}

\author{
Kyongho Kang ${ }^{1,2}$, Sukjin Yu', Geunho Lee ${ }^{2}$, and Byeong-Hwa Lee ${ }^{3 *}$ \\ ${ }^{1}$ Hyundai Rotem R\&D Center, 462-18 Sam-dong, Uiwang-shi, Kyonggi 16082, Korea \\ ${ }^{2}$ Department of Automotive Engineering, Kookmin University, 77 Jeongneung-ro, Seongbuk-gu, Seoul 02707, Korea \\ ${ }^{3}$ Korea Automotive Technology Institute, 201 Gwahakseo-ro, Dalseong-gun, Daegu 43011, Korea
}

(Received 21 July 2015, Received in final form 4 November 2015, Accepted 5 November 2015)

\begin{abstract}
This paper presents a practical method for estimation of average temperature in the permanent magnet (PM) of electric machine by using finite element analysis (FEA) and dynamo load experiment. First of all, the temperature effect of PM to the torque has been employed by FEA in order to evaluate the TemperatureTorque characteristic curve. The 1st order polynomial equation which is torque attenuation coefficient is derived by the FEA result of the Temperature-Torque curve. Next, torque saturation test with constant current condition is performed by dynamo load experiment. Then, the temperature trend can be estimated by adding the initial starting temperature using the torque attenuation coefficient and torque saturation curve. Lastly, estimated temperature is validated by infrared thermometer which measures temperature of PM surface. The comparison between the estimated result and experimental result gives a good agreement within a deviation of maximum $8^{\circ} \mathrm{C}$.
\end{abstract}

Keywords : temperature of permanent magnet, electric machine, demagnetization, permanent magnet

\section{Introduction}

Vector controlled permanent magnet synchronous machines (PMSMs) are have become widely used due to the motorization of vehicle component and an increased demand for high efficiency and high power density. In particular, PMSM made from rare earth material represented by neodymium is applied to automotive parts requiring high power density, such as hybrid and electric vehicle driving motor. Since permanent magnet (PM) based on the neodymium material has demagnetization characteristic in high temperature, residual magnetic flux density and coercive force are decreased by the increase of temperature. Therefore, in safety critical applications, degraded performance is unacceptable, and PMSM must be designed to minimize the risk of thermal demagnetization [1]. The temperature characteristic of PMSM has critical effect on the lifetime of its major components such as winding insulation, permanent magnet, and bearing [2]. The temperature of permanent magnet inside

CThe Korean Magnetics Society. All rights reserved.

*Corresponding author: Tel: +82-53-719-7873

Fax:+82-53-719-7820, e-mail: bhlee2@katech.re.kr a rotor may cause partial irreversible demagnetization due to excessive demagnetization current together with the decrease of torque according to the increase of temperature [2]. Once irreversible demagnetization is happened, the power will be reduced by irreparable physical properties of the permanent magnet even at room temperature conditions [3].

In order to estimate the PM temperature, analytical method using a thermal model and high frequency signal injection method have been tried [4-6]. Besides, in order to verify this theory, a telemetry system was proposed and calculated PM temperature was validated by a method using infrared type of temperature sensor attached to the structure [7]. The method of temperature estimation which is proposed in the previous research has a limitation for the electric machine with additional cooling system or unusual thermal source. In this case, additional thermal modeling or compensation logic is required.

This paper suggests a method of indirectly estimating an average temperature of PM inside a rotor, using the finite element analysis (FEA) in conjunction with the dynamo load experiment of PMSM generator with watercooled structure. In order to increase the accuracy of estimated rotor temperature in PMSM, material properties 
based on experiments and measured electric currents are applied as the input source for the FEA. Finally, it is intended to confirm the validity of suggested method by comparing that with the measurement results of infrared temperature sensor attached to the generator housing.

\section{Background of Proposed Method}

\subsection{Relation between output torque and PM tempera-} ture in PMSM

PMSM is classified into surface mounted PM (SPM) machine and interior PM (IPM) machine according to the structure of attaching a PM to a rotor. And this paper defined $T_{e}$, the torque of PMSM generator of IPM type, as follows eq. (1) [8].

$$
\begin{aligned}
& T_{e}=\frac{P}{2} \frac{3}{2}\left[\lambda_{P M} i_{q s}^{r}+\left(L_{d s}-L_{q s}\right) i_{d s}^{r} i_{q s}^{r}\right], \\
& P: \text { Number of poles } \\
& \lambda_{P M}: \text { Linkage flux from PM [Wb] } \\
& i_{d s}^{r}, i_{q s}^{r}: d-\text {, and } q \text {-axis current }[\mathrm{A}] \\
& L_{d s}, L_{q s}: d-\text {, and } q \text {-axis inductance }[\mathrm{H}] .
\end{aligned}
$$

The output torque of PMSM with IPM structure is defined as the sum of reluctance torque and magnetic torque. Here, in case of reluctance torque, its variation with temperature is negligible within $200^{\circ} \mathrm{C}$ because the permeability of silicone steel is almost constant at the same range [8]. Therefore, this paper is intended to consider only the effect of magnetic torque, and torque $T_{e}$ can be redefined as a function of temperature $T$. eq. (2) shows both magnetic and reluctance torque equation.

$$
T_{e}=\frac{P}{2} \frac{3}{2}\left[\lambda_{P M}(T) i_{q s}^{r}+\left(L_{d s}-L_{q s}\right) i_{d s}^{r} i_{q s}^{r}\right]
$$

\subsection{Temperature characteristic of PM}

PM materials of PMSM can be classified into rare earth materials, ceramics, and metals. Major properties of PM, on which temperature has an effect, are residual magnetic flux density (hereafter called " $B r$ ") and intrinsic coercive force (hereafter called " $i H c$ "). The $B r$ value is a property that decides the flux linkage of PMSM, and has the negative temperature coefficient that decreases flux linkage with temperature rising. The $i H c$ of PM also becomes smaller as the temperature becomes higher. Moreover, a knee point is appeared in second quadrant at the specific temperature that affects irreversible demagnetization of $\mathrm{PM}$ according to current condition.

In [8], flux linkage $\lambda_{P M}$ is proportional to the $B r$ of the PM. Thus from eq. (2), output torque also has the negative temperature coefficient with temperature. For the
Table 1. Temperature characteristic of each PM material.

\begin{tabular}{cccc}
\hline \hline Type & $\begin{array}{c}\text { Temperature } \\
\text { coefficient }\end{array}$ & Unit & Remarks \\
\hline $\mathrm{NdFeB}$ & -0.1 & $\% /{ }^{\circ} \mathrm{C}$ & Rare earth materials \\
$\mathrm{Sm}_{2} \mathrm{Co}_{17}$ & -0.025 & $\% /{ }^{\circ} \mathrm{C}$ & \\
$\mathrm{Ceramic}$ & -0.2 & $\% /{ }^{\circ} \mathrm{C}$ & Ceramics \\
Alnico 5-7 & -0.02 & $\% /{ }^{\circ} \mathrm{C}$ & Metals \\
\hline
\end{tabular}

Table 2. Major design specifications of generator.

\begin{tabular}{cc}
\hline \hline Items & Values [Unit] \\
\hline Maximum output & $120[\mathrm{~kW}]$ \\
Maximum torque & $600[\mathrm{~N} . \mathrm{m}]$ \\
No. of poles/No. of slots & $16 / 24$ \\
Winding type & Concentrated winding \\
Phase & 3 -phase \\
Stator inner/outer diameter & $380 / 258.6[\mathrm{~mm}]$ \\
Rotor inner/outer diameter & $190 / 256[\mathrm{~mm}]$ \\
Lamination stack length & $130[\mathrm{~mm}]$ \\
Rotor structure & Single-layer IPM \\
Grade of Permanent Magnet & N36Z \\
\hline
\end{tabular}

purpose of relative comparison according to magnet materials, representative temperature coefficient of $\mathrm{Br}$ is compared according to four types of PM materials as shown in Table 1.

\subsection{Method of estimating the temperature of PM}

2.3.1. Definition of analysis model

The PMSM type generator to be analyzed in this paper was designed and manufactured by three-phase IPM machine. The major design specifications were summarized in Table 2.

Figure 1 shows the assembly of generator and shape of magnetic circuit especially flux density distribution. In order to reduce the inner temperature of the machine during operation, a water-cooled housing is applied to designed generator.

\subsubsection{Method of calculation the temperature of PM}

The method of estimating the average PM temperature inside the rotor, which is presented in this paper, is a calculating method using the properties of PM and torque data obtained from a rated load test. Figure 2 shows analysis procedure for calculating the average temperature of magnet.

It is possible to assume the torque, $T_{e}$ to be eq. (3), a linear regression function of temperature, if output torque $T_{e}$ is analyzed by FEA after considering $\mathrm{Br}$ to be a function of temperature $T$.

$$
T_{e}=\alpha \cdot T+\beta,
$$




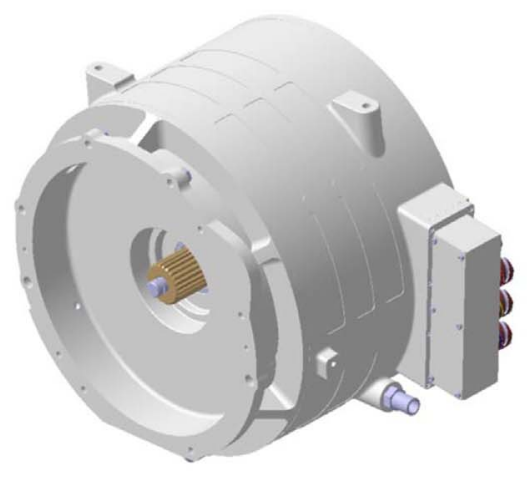

(a) Assembly of generator

Fig. 1. (Color online) Analysis model.

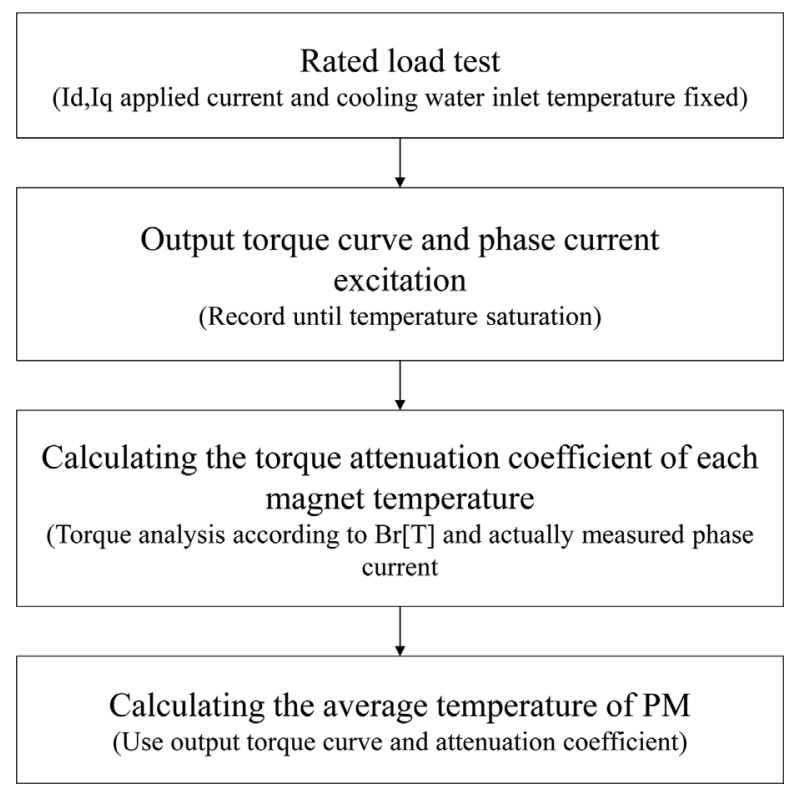

Fig. 2. (Color online) Procedure of calculating the average temperature of PM.

where $\alpha$ is torque attenuation coefficient from linear fitted equation $\left[\mathrm{N} . \mathrm{m} /{ }^{\circ} \mathrm{C}\right]$ and $\beta$ is residual torque from linear fitted equation [N.m].

If we differentiate both sides of eq. (3) with respect to time, we get eq. (4) and eq. (5).

$$
\begin{aligned}
& \Delta T_{e}=\alpha \cdot \Delta T, \\
& \Delta T=\frac{\Delta T_{e}}{\alpha} .
\end{aligned}
$$

Accordingly, it becomes possible to calculate temperature variation from actually measured torque variation.

\subsection{Experiment and analysis}

Because the developed generator is controlled by vector

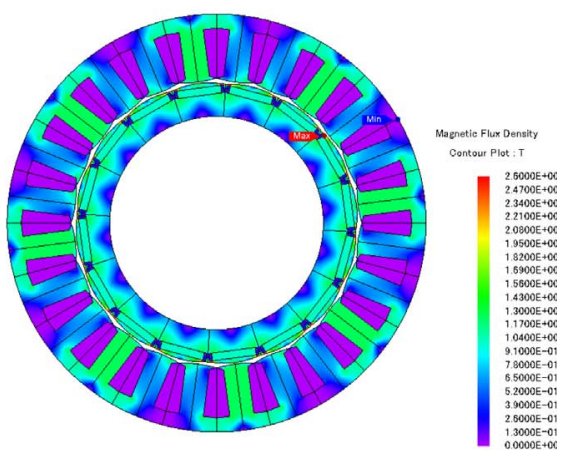

(b) Magnetic circuit

Table 3. Definition of operating points for the dynamo load experiment.

\begin{tabular}{llcc}
\hline \hline & Operating point & $\mathrm{I}_{\text {dref }}[\mathrm{A}]$ & $\mathrm{I}_{\text {qref }}[\mathrm{A}]$ \\
\hline OP1 & -393 N.m@2,500RPM & -252.2 & -75.9 \\
OP2 & -300 N.m@3,000RPM & -203.2 & -114.6 \\
\hline
\end{tabular}

control method, it is operated in dynamo load equipment with the current vector command at a set operating point. This paper is intended to estimate the average temperature of PM by using the derated toque curve and phase current waveform obtained after operating the developed generator for 2 hours with giving constant current commands at each operating point defined as shown in the following Table 3. The torque with minus sign in Table 3 means generating torque by dynamo load test evaluated in generating mode.

\subsubsection{Configuration of experiment equipment}

The developed generator was installed in test equipment for dynamo load experiment as shown in Fig. 3 so as to obtain output torque curve with the variation of PM

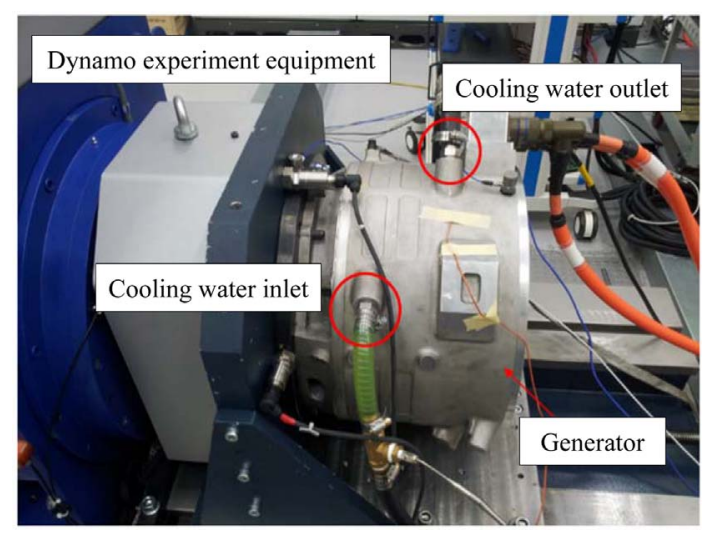

Fig. 3. (Color online) Configuration of experiment set-up. 


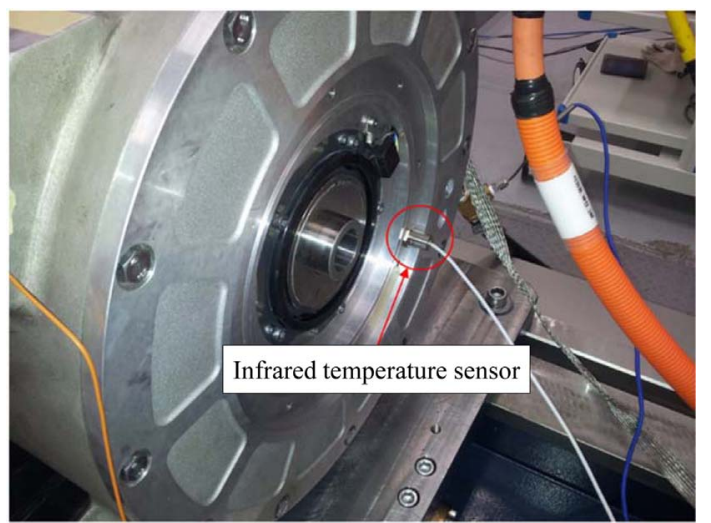

Fig. 4. (Color online) Infrared temperature sensor attachment structure.

temperature in case of a constant current command. In particular, the developed generator has a water-cooled structure. Accordingly, separated cooling equipment that is able to control the flow rate and temperature of cooling water is applied to the experimental environment. Besides, in order to compare the calculated average temperature of

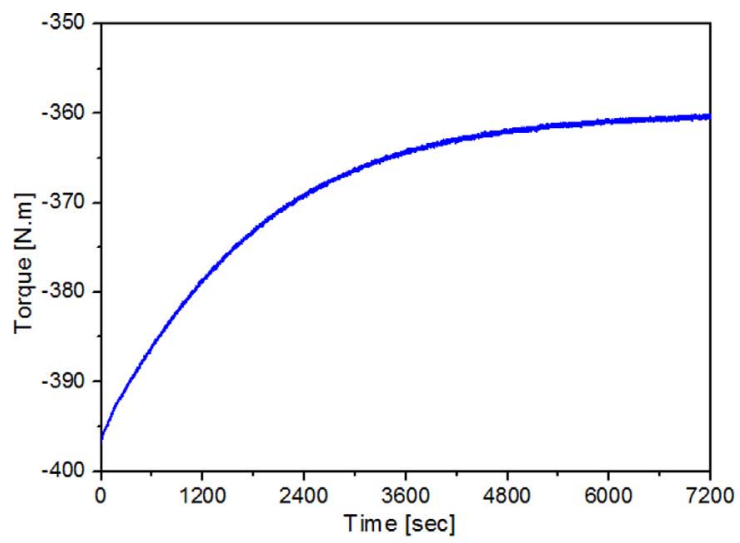

(a) Output torque curve at OP1

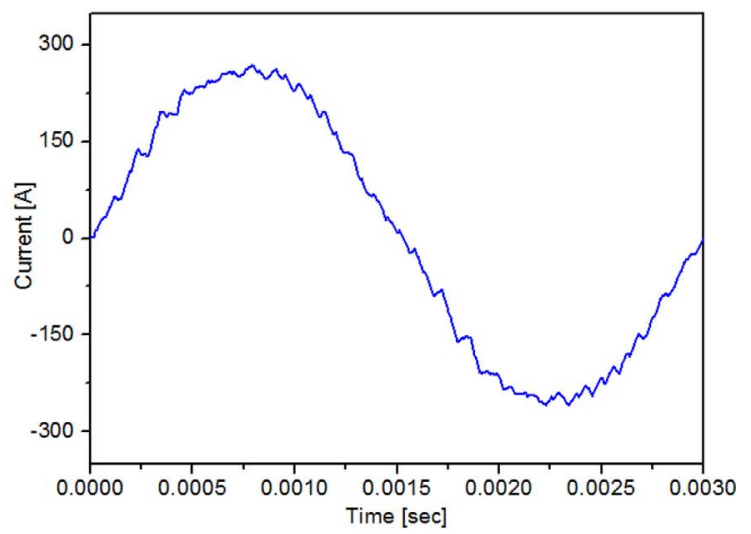

(b) Phase current waveform at OP1

Fig. 5. (Color online) Measurement results at operating point OP1.
PM with experiment results, additional infrared temperature sensor was installed outside the generator as shown in Fig. 4, and the surface temperature of rotor end plate was measured at the same time.

\subsubsection{Experimental results}

At each operating point, a dynamo rating test has been carried out for 2 hours. At this time, the temperature of cooling water inlet was fixed at $60^{\circ} \mathrm{C}$, and the flow rate of cooling water was fixed at 15LPM. The test was performed in a constant current without temperature compensation algorithm of inside of generator. An output torque curve of generator and phase current waveform at each operating point, which was obtained through the experiment, was as shown in Fig. 5 and Fig. 6, respectively.

The torque trend according to variation of temperature in PM which is shown in Fig. 7 is computed by using electromagnetic analysis software fed with measured phase current as shown in Fig. 5(b) and Fig. 6(b) as well as temperature properties of PM. The coefficient of torque variation by temperature is defined as the rate of decrease

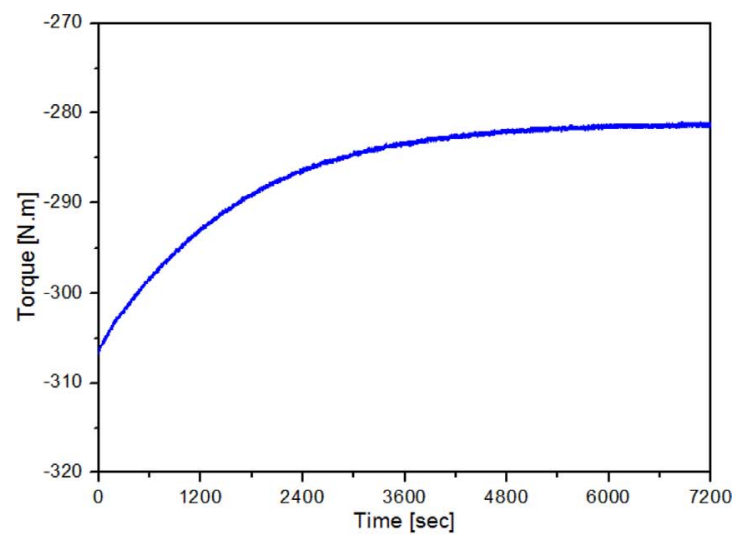

(a) Output torque curve at OP2

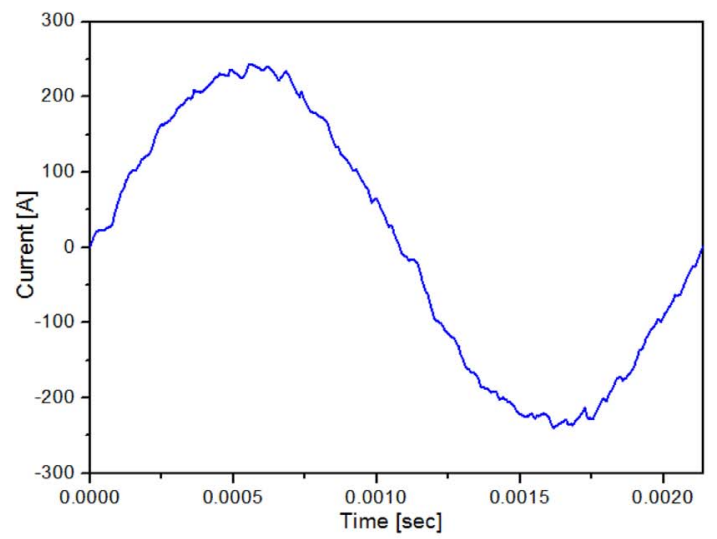

(b) Phase current waveform at OP2

Fig. 6. (Color online) Measurement results at operating point OP2. 


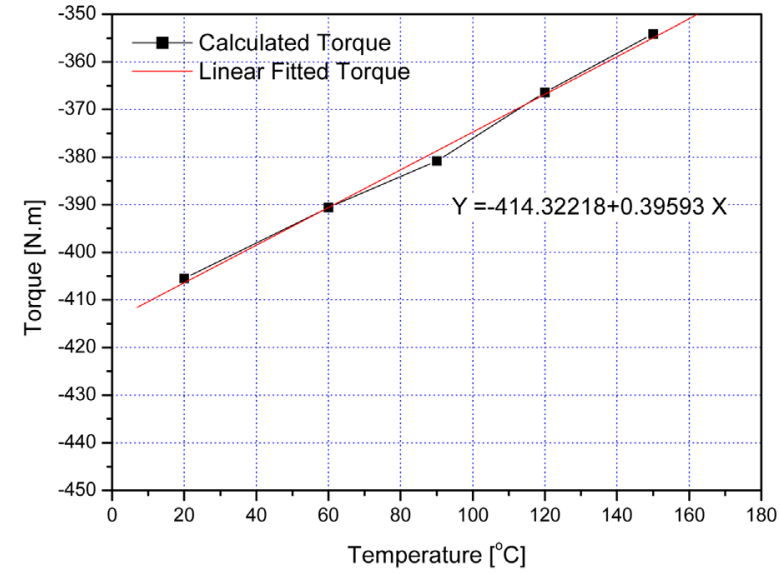

(a) Calculation of torque attenuation coefficient at OP1

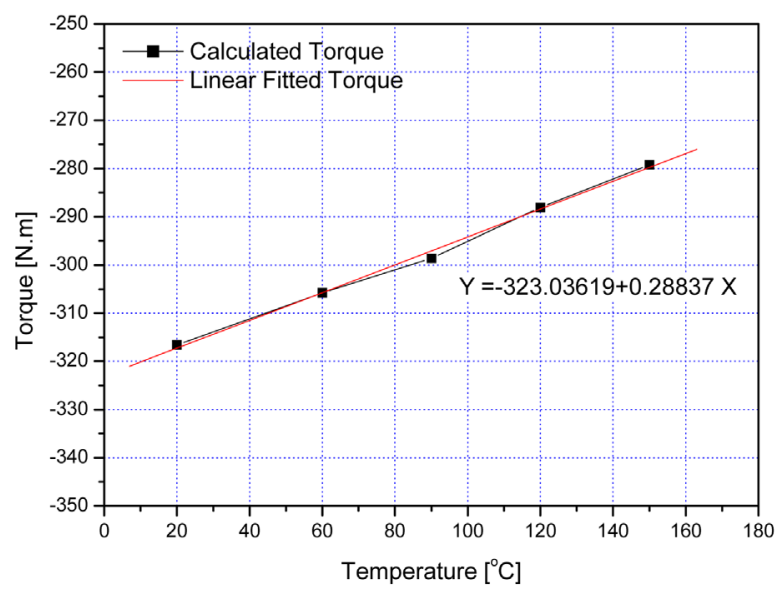

(b) Calculation of torque attenuation coefficient at OP2

Fig. 7. (Color online) Calculation of torque attenuation coefficient at each operating point.

in torque according to the PM temperature in Fig. 7. Therefore, it is expressed as a form of eq. (3) with torque attenuation coefficient.

Torque attenuation coefficient, which is calculated as shown in Fig. 7, is combined with the measured torque curve as shown in Fig. 5(a) and Fig. 6(a), it becomes possible to calculate the change of temperature by adding that to initial temperature. Therefore, the estimated magnet temperature was compared with the temperature that is measured by an infrared thermometer in Fig. 8.

\subsubsection{Analysis results}

According to the results of comparing the estimated PM temperature from a torque curve obtained through a rating test with temperature measured through an infrared sensor, there was a deviation of maximum $7.8^{\circ} \mathrm{C}$ on the basis of saturation temperature at operating point OP1, and there was a deviation of maximum $6.9^{\circ} \mathrm{C}$ at $\mathrm{OP} 2$. The

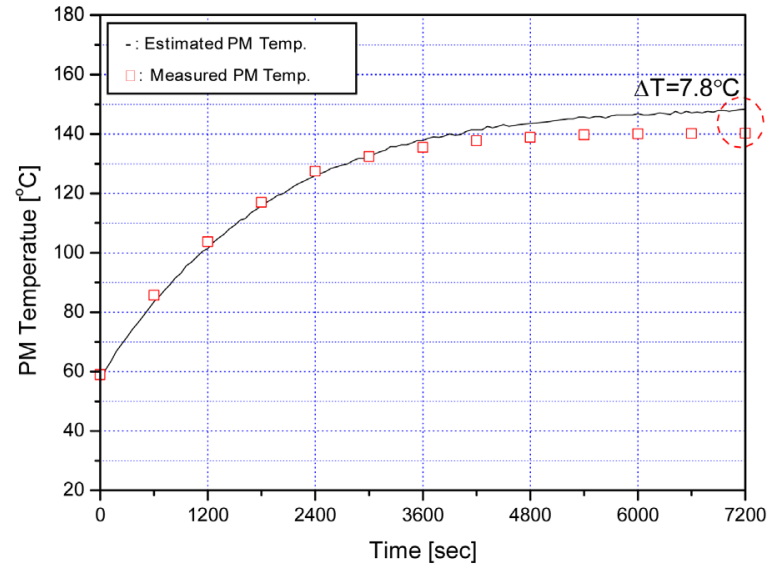

(a) The temperature of permanent magnet at OP1

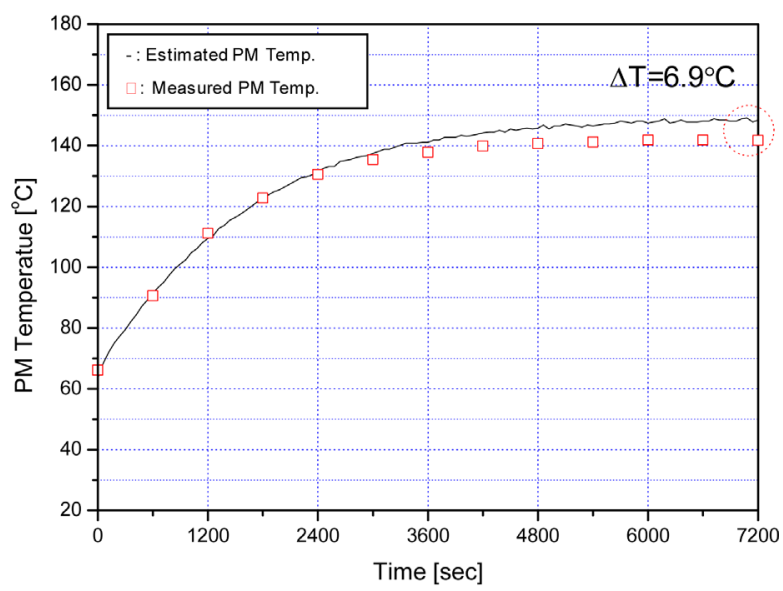

(b) The temperature of permanent magnet at OP1

Fig. 8. (Color online) Comparison between the estimated PM temperature and experiment result at each operating point.

cause of these temperature deviations can be analyzed as follows.

1) The dispersion of the material properties of the PM (mainly $\mathrm{Br}$ with temperature)

2) Deviation between actually measured and calculated torque

3) Torque sensor of dynamo-set and infrared temperature sensor measurement error

4) Local temperature deviation between the measured temperature at the measurement point of infrared temperature sensor (the surface of end plate) and estimated magnet temperature (average values)

\section{Conclusions}

In this paper, calculation method for the temperature of the PM inside a rotor core is proposed by a way to 
calculate the torque trend obtained through the dynamo experiment. Moreover, the validity of the presented method was confirmed through comparison between the magnet temperature estimated by the presented method and the value measured by using an infrared sensor in the actually manufactured $120 \mathrm{~kW}$ generator. According to the results of comparison after measurement at two operating points, a maximum error of $7.8^{\circ} \mathrm{C}$ was confirmed. The suggested method estimates the temperature of magnet by using the results of dynamo performance test that shall be essentially performed at a stage of developing the product of PMSM. Accordingly, the test duration can be reduced without additional measurement equipment, and relatively accurate temperature can be estimated even in the environment where it is difficult to install a telemetry system or infrared sensor. In the future, with regard to the major causes of temperature deviation, which have been determined by the analysis, we plan to make a sensitivity analysis, and plan to additionally examine a compensation method that can reduce the deviation.

\section{References}

[1] James D. Mcfarland, and T. M. Jahns, IEEE Trans. Indus. Applications 50, 2768 (2013).

[2] R. Ruoho, J. Kolehmainen, J. Ikaheimo, and A. Arkkio, IEEE Trans. Magn. 46, 949 (2010).

[3] R. R. Errabelli and P. Mutschler, IEEE Trans. Power Electron. 27, 500 (2012).

[4] C. Kral, A. Haumer, and Lee Sang Bin, IEEE Trans. Power Electronics 29, 455 (2014).

[5] S. Stipetic, M. Kovacic, Z. Hanic, and M. Vrazic, IEEE Trans. Ind. Electron. 59, 2288 (2012).

[6] D. D. Reigosa, F. Briz, P. Garcia, J. M. Guerrero, and M. W. Degner, IEEE Trans. Ind. Appl. 46, 1468 (2010).

[7] M. Ganchev, H. Umschaden, and H. Kappeler, Proc. IEEE $37^{\text {th }}$ Annu. Conf. Ind. Electron. Soc., 2011 (2006).

[8] Yeonsu Kim, Ph.D. Thesis, Seoul National University, Korea (2007). 